\title{
Testing multiple polynomial models for eye-tracker calibration
}

\author{
Carlos Lara-Alvarez ${ }^{1}$ (D) . Fernando Gonzalez-Herrera ${ }^{2}$
}

Received: 23 August 2019 / Accepted: 28 January 2020 / Published online: 28 May 2020

(C) The Psychonomic Society, Inc. 2020

\begin{abstract}
The straightforward approach to eye-tracker calibration considers that the calibration data do not have erroneous associations, and the calibration function is defined. The violation of the non-erroneous assumption could cause an arbitrarily large bias. The MMransac algorithm proposed in this paper is a modified version of the Random Sample Consensus. that achieves robust calibrations. On the other hand, polynomials in two variables (i.e., with terms in the form $\kappa x^{a} y^{b}$ ) are commonly used to map eye-tracker measurements to points on the screen. High-degree polynomials tend to be more accurate; however, as the order is increased, the function becomes more complex and less smooth, which could cause overfitting. In this sense, this paper proposes an algorithmic approach that enables model selection criteria even in the presence of outliers. This approach was tested using different model selection criteria. Results show that more accurate calibrations are obtained with the combined robust fitting and model selection approach using the Akaike information criterion (AIC) and the Kullback information criterion (KIC).
\end{abstract}

Keywords Eye tracking $\cdot$ Calibration $\cdot$ Akaike information criterion

\section{Introduction}

Eye trackers provide information of eye behavior, such as the location and duration of eye-made events. These events can provide insight of human behavior in areas such as: human-computer interaction, medicine, psychology, advertisement, and other research fields. Eyes make intentional and unintentional movements that can be measured by hardware and translated into signals to later be mapped into screen coordinates (Majaranta \& Bulling, 2014).

Eye trackers rely on the calibration stage to calculate accurate screen coordinates; for instance, up to $20 \%$ of

Carlos Lara-Alvarez

c.alberto.lara@gmail.com

Fernando Gonzalez-Herrera

jfernandoghe@gmail.com

1 Information Technology Laboratory, Center for Research and Advanced Studies, Parque Cientifico y Tecnologico Tecnotam Km, 5.5 carretera Cd. Victoria - Soto La Marina C.P., 87130, Cd. Victoria, Tamaulipas, Mexico

2 Laboratory of Human-Centered Computing, Center of Mathematical Research (CIMAT), Calle Lasec y Andador Galileo Galilei, Quantum, Ciudad del Conocimiento C.P. 98160, Zacatecas, Mexico experiments in ET studies need re-calibration due to poor performance (Goldberg \& Wichansky, 2003) because a wrong calibration makes the information unusable. Measurements are mapped to computer coordinates by using different techniques (Martinez et al., 2012); however, regression using least squares is widely because of its low computational cost and acceptable accuracy. Different regression models (functions) can modify the performance of the calibration; hence, a reliable and simple model is required (Kasprowski et al., 2014).

The contributions of this paper are: i) a sample consensus approach to obtain a robust and more accurate calibration, and ii) an approach that enables using different statistics to model selection.

The rest of this paper is organized as follows: Section "Related work" reviews approaches proposed in the literature to eye-tracking calibration. Section "Background" reviews the regression models and model selection. Section "Proposed approach (MMrANSAC)" describes the proposed approach. Section "Materials and methods" describes the experiments. Section "Results and discussion" discuses and analyzes the results. Finally, Section "Conclusions and further work" presents conclusions and proposals for future work.

In order to simplify the lecture of this paper, a list of abbreviations and symbols that are used in the paper is given in Table 1. 
Table 1 List of different abbreviations and symbols

\begin{tabular}{ll}
\hline$x, y$ & uncorrected coordinates \\
$\check{x}, \check{y}$ & corrected coordinates \\
$x^{\prime}, y^{\prime}$ & screen coordinates \\
$F(x, y)$ & $\begin{array}{l}\text { Polynomial vector of bivariate } \\
\text { terms in the form } x^{a} y^{b} \quad a, b \in\end{array}$ \\
& $\mathbb{N}$ \\
$M_{r}$ & $r$-th model to correct $x$ \\
$N_{s}$ & $s$-th model to correct $y$ \\
$\mathbf{m}, \mathbf{n}$ & coefficients for models $M$ and $N$, respectively.
\end{tabular}

\section{Related work}

The main concern with gaze calibration is to guarantee that the user is effectively seeing a determined calibration point. For this aim, Interval Selection with Median Filter (ISMF) first removes initial measurements (when saccades happen more likely) and then the gaze position is computed as the median value of the data collected during the considered period (Gibaldi et al., 2017). This assumption cannot be justified in some situations; e.g., for eye-tracking studies with infants. Measurements wrongly associated with calibration points could cause a significant bias in the parameter estimation. In this paper, an extension was made to the Random Sample Consensus (RANSAC) method (Fischler \& Bolles, 1981) to obtain a good fitting model even when the user did not observe one or more calibration points.

In the common approach to calibrate an eye tracker, the user must observe a pattern of predefined points on the screen; Fig. 1 shows calibration patterns of 3, 5, 9, and 13 points. A calibration point is obtained by associating a point on the screen to a set of measured points, then the parameters of a function (or model) that maps measured to screen points are selected. Common calibration models are homographies (Hansen et al., 2010), polynomial relations (Zhu et al. 2007; Cherif et al., 2002), SVR (Krafka et al., 2016), and neural networks (Pomplun et al., 1994).

Blignaut (2016) considers that it is worth spending more time in the ET calibration to obtain better results, but, in general, this procedure is considered tedious and time consuming for the user (Zhu \& Ji, 2005; Ohno \& Mukawa, 2004) as it requires at least the same number of calibrations
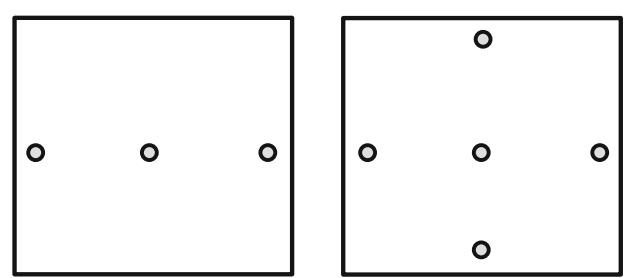

Table 2 Polynomial models with less than or equal to four parameters

\begin{tabular}{|c|c|c|c|}
\hline \multicolumn{4}{|l|}{$x$-axis } \\
\hline Model & $F_{x}(x, y)$ & $k_{M_{r}}$ & Used in \\
\hline$M_{1}$ & {$[x, 1]$} & 2 & Blignaut (2016) \\
\hline$M_{2}$ & {$[x, y, 1]$} & 3 & Blignaut (2016) \\
\hline$M_{3}$ & {$[x, x y, 1]$} & 3 & Blignaut (2016) \\
\hline$M_{4}$ & {$\left[x^{2}, x, 1\right]$} & 3 & Blignaut (2016) \\
\hline$M_{5}$ & {$\left[x^{3}, x, 1\right]$} & 3 & Blignaut (2016) \\
\hline$M_{6}$ & {$[x, y, x y, 1]$} & 4 & $\begin{array}{l}\text { Hennessey et al. (2008) and Zhu } \\
\text { and Ji (2005) }\end{array}$ \\
\hline$M_{7}$ & {$\left[x^{3}, x^{2}, x, 1\right]$} & 4 & Blignaut (2016) \\
\hline$M_{8}$ & {$\left[x^{2}, x, y, 1\right]$} & 4 & Cerrolaza et al. (2008) \\
\hline$M_{9}$ & {$\left[x^{2}, x, x y, 1\right]$} & 4 & Blignaut (2016) \\
\hline$M_{10}$ & {$\left[x^{3}, x, y, 1\right]$} & 4 & Blignaut (2016) \\
\hline$M_{11}$ & {$\left[x^{3}, x, y^{2}, 1\right]$} & 4 & Blignaut (2016) \\
\hline$M_{12}$ & {$\left[x^{3}, x, x y, 1\right]$} & 4 & Blignaut (2016) \\
\hline \multicolumn{4}{|l|}{$y$-xis } \\
\hline Model & $F_{y}(x, y)$ & $\mathrm{k}$ & Used in \\
\hline$N_{1}$ & {$[y, 1]$} & 2 & Blignaut (2016) \\
\hline$N_{2}$ & {$[y, x, 1]$} & 3 & Blignaut (2016) \\
\hline$N_{3}$ & {$[y, x y, 1]$} & 3 & Blignaut (2016) \\
\hline$N_{4}$ & {$\left[y^{2}, y, 1\right]$} & 3 & Blignaut (2016) \\
\hline$N_{5}$ & {$\left[y, x^{2}, 1\right]$} & 3 & Blignaut (2016) \\
\hline$N_{6}$ & {$[y, x y, x, 1]$} & 4 & Hennessey et al. (2008) \\
\hline$N_{7}$ & {$\left[y^{2}, y, x, 1\right]$} & 4 & Blignaut (2016) \\
\hline$N_{8}$ & {$\left[x y^{2}, x, y, 1\right]$} & 4 & Cerrolaza et al. (2008) \\
\hline$N_{9}$ & {$\left[x y, y, x^{2}, 1\right]$} & 4 & Blignaut (2016) \\
\hline$N_{10}$ & {$\left[x^{2} y, x^{2}, y, 1\right]$} & 4 & Blignaut (2016) \\
\hline
\end{tabular}

points as the number of parameters; i.e., a five-parameter model requires five calibration points, a model with nine parameters requires nine points, and so on.

In two variables, a polynomial function is a linear combination of a finite number of terms (monomials) in the form $x^{a} y^{b}$. The domain of any polynomial function of two variables is the whole plane. Different polynomials have been used as models for ET calibration. Table 2 summarizes polynomial models, $F_{x}$ and $F_{y}$, with less than or equal to four parameters.

Several authors have compared polynomial models. For instance, Blignaut (2016) compares different correction

Fig. 1 Common patterns for initial calibration 
models for a 45-point calibration pattern, focusing on selecting the best polynomial per participant.

Cerrolaza et al. (2008) test models with up to 16 terms (with a long and tedious calibration stage). Their results show that second-degree models can perform similar to more complex models, with insignificant differences in the range of 1-2 pixels on a screen with resolution of $1024 \times 768$.

Kasprowski et al. (2014) use linear, quadratic, and cubic polynomials with 3,5 , and 18 calibration points, respectively. They proposed discarding models based on the ratio of the sum of squares residuals to the sample variance.

Kasprowski et al. (2014) compare calibration results for two different head mounted eye trackers by using cross validation, root mean error, coefficient $R$, and square and absolute error. Their results show that the optimal degree for the polynomial calibration function depends on the specific ET device.

In contrast to the previous work, this paper studies calibration with few points and possibly with outliers, and it proposes a statistical criterion based on the 'parsimony principle' to obtain the best model.

\section{Background}

For completeness, this section outlines some background materials on regression and statistical tools that apply the parsimony principle for model selection.

\section{Regression}

Polynomials in two variables can be written as

$F(x, y) \cdot \mathbf{a}$

where $F(x, y)$ is a vector with terms in the form $x^{a} y^{b}$ $a, b \in \mathbb{N}$, $\mathbf{a}$ is a vector of coefficients, and "." denotes the scalar product of the vectors.

To calibrate a gaze point, the following functions are used:

$\check{x}=F_{x}(x, y) \cdot \mathbf{m}=M \cdot \mathbf{m}$

$\check{y}=F_{y}(x, y) \cdot \mathbf{n}=N \cdot \mathbf{n}$

where $x, y$ are uncorrected coordinates given by the eye tracker, $\check{x}, \check{y}$ are corrected coordinates, and $\mathbf{m}$, $\mathbf{n}$ are the parameters of the polynomial model.

Henceforth, the discussion focuses in the $x$-axis calibration, but this description also holds for $y$-axis calibration.

Given $m_{p}$ calibration pairs: $\left(x_{1}^{\prime}, y_{1}^{\prime}\right) \mapsto\left(x_{1}, y_{1}\right), \ldots$, $\left(x_{n}^{\prime}, y_{n_{p}}^{\prime}\right) \mapsto\left(x_{n}, y_{m_{p}}\right)$, and a given model $M=F_{x}(x, y)$. The coefficients $\hat{\mathbf{m}}$ can be estimated as:

$\hat{\mathbf{m}}=\left(X X^{\top}\right)^{-1} X^{\top} \mathbf{x}$ where $X$ is the matrix whose $i$-th row is $F_{x}\left(x_{i}^{\prime}, y_{i}^{\prime}\right)$ and $\mathbf{x}=\left[x_{1}, x_{2}, \ldots, x_{m_{p}}\right]^{\top}$.

\section{Model selection}

The parsimony principle-aka Occam's, Ockham's or Ocham's razor principle-states that the simplest solution tends to be the correct one. In least-squares model selection, this translates as looking for the simplest solution that closely resembles the true model. This approach uses heuristics, which in essence deliver a good performance in most of the cases. Although simpler models are preferred, this principle is a trade-off between model fit and complexity.

Different approaches for model selection can be found in the literature, with wide applications and criteria. Bayesian information criterion (BIC) and Akaike information criterion (AIC) are widely used on different research areas. They penalize models with many parameters to ensure no overfitting; while still looking for the minimum error. The key difference between these two criteria is that BIC considers the true model is tested while AIC does not (Burnham \& Anderson, 2003).

The AIC can be stated as:

$\mathrm{AIC}=2 k-2 \ln (\hat{l})$,

where $\hat{l}$ is the likelihood function, and $k$ is the number of estimated parameters in the model. The model having the smallest AIC value is usually selected. In essence, the AIC prefers the overall simplest model with maximum likelihood.

For least squares approximations with independent identical normal distributions, Eq. 4 can be stated as:

$\mathrm{AIC}=2 k+n \ln \left(\frac{\mathrm{RSS}}{n}\right)$,

where $n$ is the sample size, and RSS is the residual sum of squares.

For a relatively small number of experimental points, corrections must be introduced in Eq. 4. The corrected AIC (AICc) is a special case of AIC for few samples that prevents overfitting (Cavanaugh \& et al. 1997),

$\mathrm{AICc}=\mathrm{AIC}+\frac{2 k^{2}+2 k}{n-k-1}$,

where $k$ is the number of parameters, and $n$ is the sample size; hence, for many samples $(n \rightarrow \infty)$, the AICc converges to AIC.

Other metrics have been proposed to decide on the best model against a finite model set. Some of these metrics are: the robust AIC (RAIC), the Kullback information criterion 
(KIC), and the corrected KIC (KICc). They are defined as (Cetin \& Erar, 2002; Seghouane \& Bekara, 2004):

$$
\begin{aligned}
\operatorname{RAIC}(p, \alpha, \rho)= & 2 \sum \rho\left(r_{i, P}\right)+\alpha \rho \\
\mathrm{KIC}= & -2 \ln (\hat{l})+3(k+1) \\
\mathrm{KICc}= & -2 \ln (\hat{l})+2 \frac{(k+1)}{(n-k-2)} \\
& -n \Phi\left(\frac{n-k}{2}\right)+\ln \frac{n}{2}
\end{aligned}
$$

where $\hat{\sigma}$ is a robust estimate of $\sigma ; \alpha=2 ; \rho_{c}$ is the Huber's function,

$\rho_{c}(r)= \begin{cases}\frac{r^{2}}{2}, & \text { if }|r| \leq c \\ c|r|^{2}-\frac{c^{2}}{2}, & \text { otherwise. }\end{cases}$

and $\Phi$ is the digamma function (Bernardo \& et al. 1976).

\section{Proposed approach (MMRANSAC)}

The RANSAC is an iterative method to estimate parameters of a model from a set of observed data that contains outliers (Fischler \& Bolles, 1981; Raguram et al., 2008). The random sample paradigm does that by randomly drawing minimal sets from the observed data and constructing models; i.e., assigning values for each coefficient in the model (Fischler \& Bolles, 1981). A minimal set is the smallest number of points required to uniquely define a given model. The resulting candidate coefficients are tested against all points in the data to determine how many of the points (inliers) are well approximated by the model. After a given number of trials, the model which approximates the most points is extracted. Finally, the resulting set of inliers are used to improve the model.

The proposed approach (Algorithm 1), named Multimodel RANSAC or MM-RANSAC, follows the random sample consensus paradigm. The conventional RANSAC approach tests a single model by uniformly picking a random minimal set, and using the consensus to extract the best model. In contrast, the MMRANSAC tests multiple models by: i) generating minimal samples in such way that each sample contains measurements from different calibration points, ii) using a likelihood function to test every model, and iii) using a model selection criterion to obtain the best model.

\section{Likelihood estimation}

Let $\hat{x}=\left[\hat{x}_{1}, \ldots, \hat{x}_{n}\right]$ be a vector of coordinates corrected with a polynomial model with coefficients $\mathbf{m}$, and $c_{i}$ be the indicator variable taking the value of 1 if the $\hat{x}_{i}$ is an inlier and $c_{i}=0$ otherwise. Assuming Gaussian measurement noise, the inlier's residual distribution is,

$p\left(\hat{x}_{i} \mid c_{i}=1\right)=\frac{1}{\sqrt{2 \pi \hat{\sigma}^{2}}} e^{-\frac{1}{2} \frac{\left(\hat{x}_{i}-x_{i}^{\prime}\right)^{2}}{\hat{\sigma}^{2}}}$

where $\hat{\sigma}^{2}=\left[\sum_{i} c_{i}\left(\hat{x}_{i}-x_{i}^{\prime}\right)^{2}\right] / n_{i}=\operatorname{RSS}_{i} / n_{i}$ is the maximum likelihood estimate for the variance.

As proposed by Liu et al. (2001), in different situations, outliers can be modeled as a uniform distribution. Suppose that during the calibration process, the user observes at any point on the screen with the same probability; then, the outlier's distribution is

$$
\begin{aligned}
p\left(\hat{x}_{i} \mid c_{i}=0\right) & =\frac{1}{x_{\max }} \\
& =\frac{1}{\sqrt{2 \pi \hat{\sigma}^{2}}} e^{-\frac{1}{2} \ln \frac{x_{\max }^{2}}{2 \pi \hat{\sigma}^{2}}}
\end{aligned}
$$

where $x_{\max }$ is the upper limit of the range of corrected values. Combining (11) and (12),

$p\left(\hat{x}_{i} \mid \mathbf{m}\right)=\frac{1}{\sqrt{2 \pi \hat{\sigma}^{2}}} e^{-\frac{1}{2}\left[c_{i} \frac{\left(\hat{x}_{i}-x_{i}^{\prime}\right)^{2}}{\hat{\sigma}^{2}}+\bar{c}_{i} \ln \frac{x_{\mathrm{max}}^{2}}{2 \pi \hat{\sigma}^{2}}\right]}$

Assuming independence in measurement noise, the joint probability is

$p(\hat{x} \mid \mathbf{m})=b\left(n_{i}\right) \prod \frac{1}{\sqrt{2 \pi \hat{\sigma}^{2}}} e^{-\frac{1}{2}\left[c_{i} \frac{\left(\hat{x}_{i}-x_{i}^{\prime}\right)^{2}}{\hat{\sigma}^{2}}+\bar{c}_{i} \ln \frac{x_{\max }^{2}}{2 \pi \hat{\sigma}^{2}}\right]}$

and the $\log$ likelihood, $\hat{L}(\mathbf{m})=\ln \hat{l}(\mathbf{m})$, is

$$
\begin{array}{r}
\hat{L}(\mathbf{m})=\ln b\left(n_{i}\right)+n \ln \frac{1}{\sqrt{2 \pi \hat{\sigma}^{2}}}-\frac{1}{2} \sum_{i} c_{i} \frac{\left(\hat{x}_{i}-x_{i}^{\prime}\right)^{2}}{\hat{\sigma}^{2}} \\
-\frac{n_{o}}{2} \ln \frac{x_{\max }^{2}}{2 \pi \hat{\sigma}^{2}} \\
=\ln b\left(n_{i}\right)-\frac{1}{2} n_{i}\left(\ln \hat{\sigma}^{2}+1\right)-\frac{1}{2} n_{o}\left(\ln x_{\max }^{2}-\ln 2 \pi\right) \\
+C
\end{array}
$$

where $n_{i}, n_{o}$ are the number of inliers and outliers, respectively; $b\left(n_{i}\right)$ is the binomial probability of $n_{i}$ successes in $n$ trials with success probability $p_{\text {in }}$; and $C$ is a constant. To prevent numerical problems of Eq. 16 we set $\hat{\sigma}$ to one, for estimated values below one.

\section{Algorithm description}

Let $\mathcal{D}$ be the calibration set, $\mathcal{D}_{1}, \ldots \mathcal{D}_{n}$ be a partition of $\mathcal{D}$ and $k_{r} \leq n$ be the number of parameters required for the $r$-th model, $M_{r}$. Each subset $\mathcal{D}_{i}$ contains measurements generated when the user observes the $i$-th calibration point of a pattern of $n$ points. The robust fitting algorithm (Algorithm 2), first randomly selects $k_{r}$ different subsets from $\mathcal{D}_{1}, \ldots \mathcal{D}_{n}$; then, a single point is selected randomly 
from each subset to obtain a minimal set. 3). A graphical illustration of the proposed selection approach is shown in Fig. 2.

The parameters $\mathbf{m}$, generated from a minimal set, are tested against $\mathcal{D}$ with Eq. 16 by taking the corrected measurement $\hat{x}_{i} \in \mathcal{D}_{i}$ that gives some $q$-quantile residual.

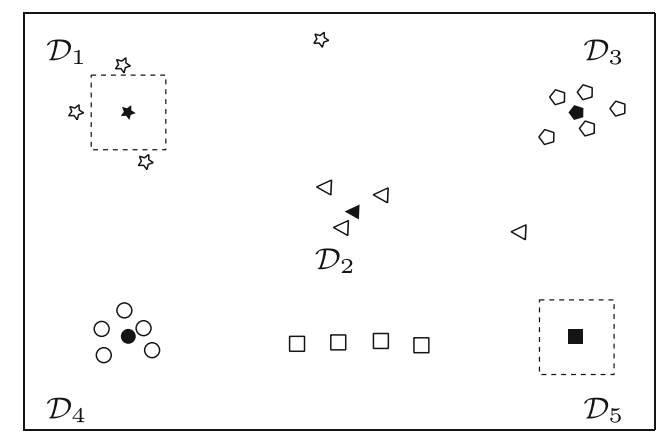

(a) $i$-iteration (subset selection)

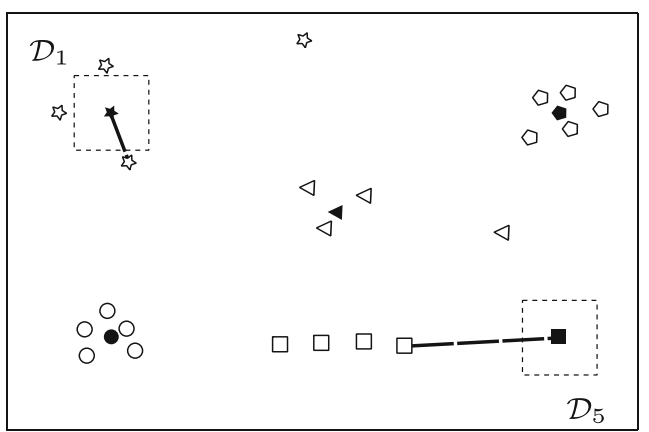

(b) $i$-th iteration (measurement selection)

:

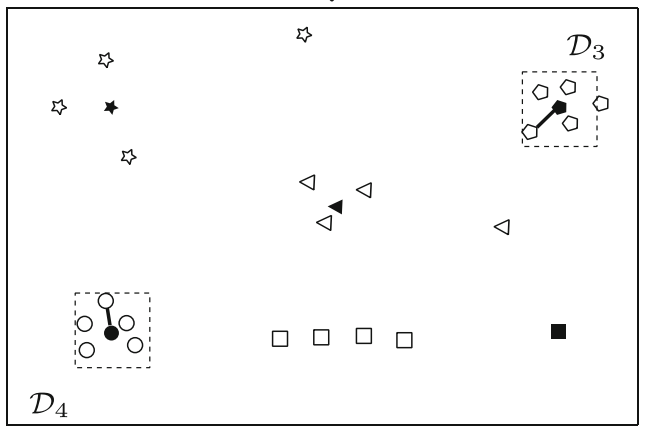

(c) $j$-th iteration (best model)

Fig. 2 MMransAC (Selection step). Given a calibration set, $\mathcal{D}$, composed of $\mathcal{D}_{1}$ (stars), $\mathcal{D}_{2}$ (triangles), $\mathcal{D}_{3}$ (pentagons), $\mathcal{D}_{4}$ (circles), and $\mathcal{D}_{5}$ (squares), and a model uniquely defined by two points: a Subset selection. the algorithm selects $\mathcal{D}_{1}$ and $\mathcal{D}_{5}$ in some iteration, b Measurement selection. A single measurement is selected for each partition, after this step we have a minimum set, then the model is defined. The likelihood for the model generated is low because $\mathcal{D}_{5}$ does not have true measurements, $\mathbf{c}$ the process-subset and measurement selection-is repeated until a proper model is found; in this example, the model generated from $\mathcal{D}_{3}$ and $\mathcal{D}_{4}$ have a maximum consensus
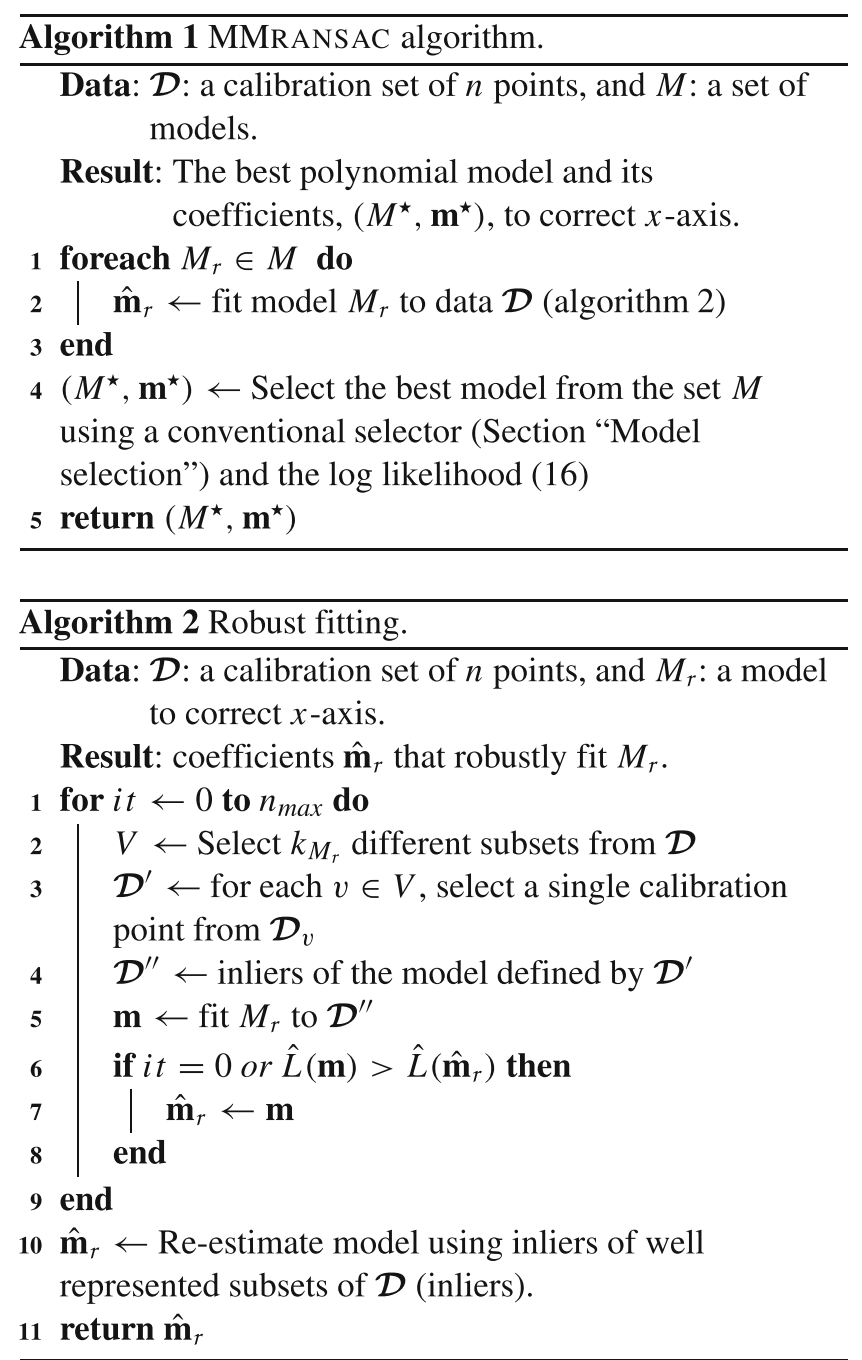

Consider the example shown in Fig. 2. We expect that the model $\mathbf{m}_{1}$ generated from $\left\{\mathcal{D}_{1}, \mathcal{D}_{5}\right\}$ has a lower loglikelihood than the model $\mathbf{m}_{2}$ generated from $\left\{\mathcal{D}_{4}, \mathcal{D}_{3}\right\}$. In normal conditions and considering the lower residual as the representative point, the first model could have two inliers and three outliers $\left(n_{i}=2, n_{o}=3\right.$, and $\left.\hat{\sigma}=\sigma_{\min }=1\right)$; on the other hand, $\mathbf{m}_{2}$ could have four inliers and one outlier $\left(n_{i}=4, n_{o}=1\right.$, and $\left.\hat{\sigma} \leq \tau\right)$ where $\tau$ is the threshold used for inlier/outlier labeling. Applying Eq. 16, the $\mathbf{m}_{2}$ is preferred over $\mathbf{m}_{1}$ when

$\ln b(4)+\ln x_{\max }^{2}>\ln b(2)+\ln \tau^{2}+1+\ln 2 \pi$

which is typically true as we expect a low proportion of outliers $\ln b(4)>\ln b(2)$, and the screen value is much greater than the threshold, $x_{\max } \gg \tau$.

The log likelihood also enables using selection criteria to select a single model. In this sense, Eq. 16 is replaced in Eqs. 4, 6, 8, and 9 to select the best model in noise points. This is the final step in Algorithm 1 (line 4). 
Table 3 Information summary from the 'calibration data' structure in the PD dataset (Drewes et al., 2014)

\begin{tabular}{ll}
\hline Field(s) & Description \\
\hline Subject & Subject number \\
Repeat & Repetition number \\
Luminance & Background luminance, $\{0,12.5, \ldots\}$ \\
Point & Calibration point number $\{1, \ldots, 25\}$ \\
screenX, & Reference screen coordinates of a calibra- \\
screenY & tion point, $x^{\prime}$ and $y^{\prime}$, respectively \\
Epoch & Raw points measurements, $\left[(L, R)_{t}\right]_{t=1}^{f}$ \\
& from the eyetracker. Where $L$ and $R$ \\
& are triplets for the left and right eye, \\
& composed of uncorrected coordinates and \\
& the distance to the corresponding eye. \\
\hline
\end{tabular}

\section{Materials and methods}

Dataset The 'Pupil Dynamics' (PD) dataset (Drewes et al., 2014), contains eye-tracking measurements (fixation samples) from 39 subjects in a real-world laboratory settings; the Eyelink ${ }^{\circledast} 1000$ hardware (SR Research Ltd. 2010) in "desktop" configuration was used to track both eyes of the subjects at $500 \mathrm{~Hz}$ sampling frequency, with a Samsung 22 in LCD display or a ViewSonic 20 in CRT display at approximately $60 \mathrm{~cm}$ viewing distance. A 25 point pattern was used to obtain gaze points as well as screen locations; i.e., each gaze point is associated to a calibration point on the screen.

The PD dataset was selected primarily because the built-in calibration mechanism of the eye tracker was not utilized, and raw, uncalibrated points are provided to derive calibration solutions manually.

This dataset contains calibration data for different background luminances $(0,12.5,25,37.5,50,75$ and $100 \%$ of the available screen luminance) to produce measurements with both constricted and dilated pupil, as well as intermediate pupil sizes. Drewes et al. (2014) repeated the calibration procedure three times. The dataset also contains information for the two eyes. In the following experiments, a calibration set was considered for every combination of participant, eye, trial, and luminance. The original dataset contains data from two phases (calibration and fixation); here, just the data from the calibration phase is used. Table 3 summarizes the information provided by the 'calibration data'.

Another dataset, henceforth called Synthetic Random Outlier (SRO) dataset, was generated from the PD dataset but injecting an outlier. Every calibration set of the SRO has eight out of nine subsets (calibration points) of the original PD; but a single random point generated adding a noise uniformly on $(\delta,-\delta)$ to the original screen coordinate, where $\delta$ is a constant value that corresponds to $10 \%$ of the maximum coordinate.
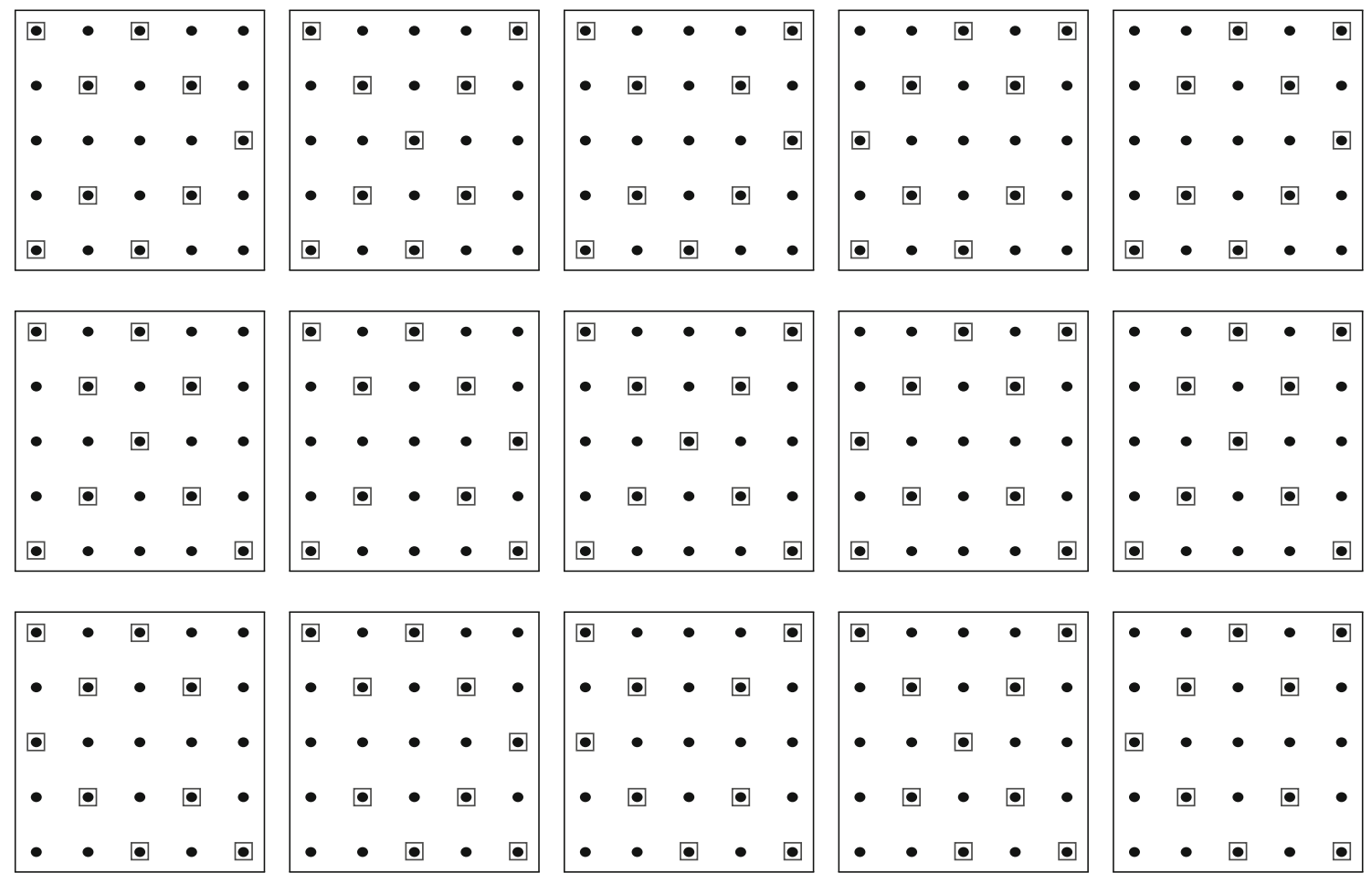

Fig. 3 Calibration patterns used in tests. Squared points indicate calibration subsets. The remaining subsets are used for testing 
Preprocessing data To accelerate the computations, a preprocessing stage in which a sample of $N / 30$ gaze measurements were selected randomly without replacement from the set of $N$ gaze measurements obtained by a single participant. After this step, measurements in the sample are in the same order that they appear in the original measurements.

Calibration / testing points The calibration pattern used in Drewes et al. (2014) consists of 25 points. In the experiments presented here, each calibration set was divided into calibration and testing points using each pattern shown in Fig. 3. The selected patterns are composed of four corners of a centered-square of $3 \times 3$ points. They also have a single calibration point in the middle row, and at most two points for each row and column. The rest of the points (16) were used for testing purposes.

Models and estimation strategies Polynomial models shown in Table 2 were used in the tests. Four approaches to estimate the parameters of polynomial models were compared: a) Simple, that uses (3) with all the pairs after the subsampling stage, b) ISMF, that removes initial measurements and takes a median value of the remaining points to get each calibration pair, the conventional RANSAC approach, and c) MMRANSAC, proposed in this paper with a success probability of $p_{\text {in }}=0.9$. For the RANSAC and MMRANSAC strategies, we set a threshold, $\tau$ equals to an angular difference of 2 degrees which is almost four times the reported accuracy for the EyeLink, but enough to reject outliers.

Table 4 Summary statistics for the first quartile angular error (expressed in degrees)

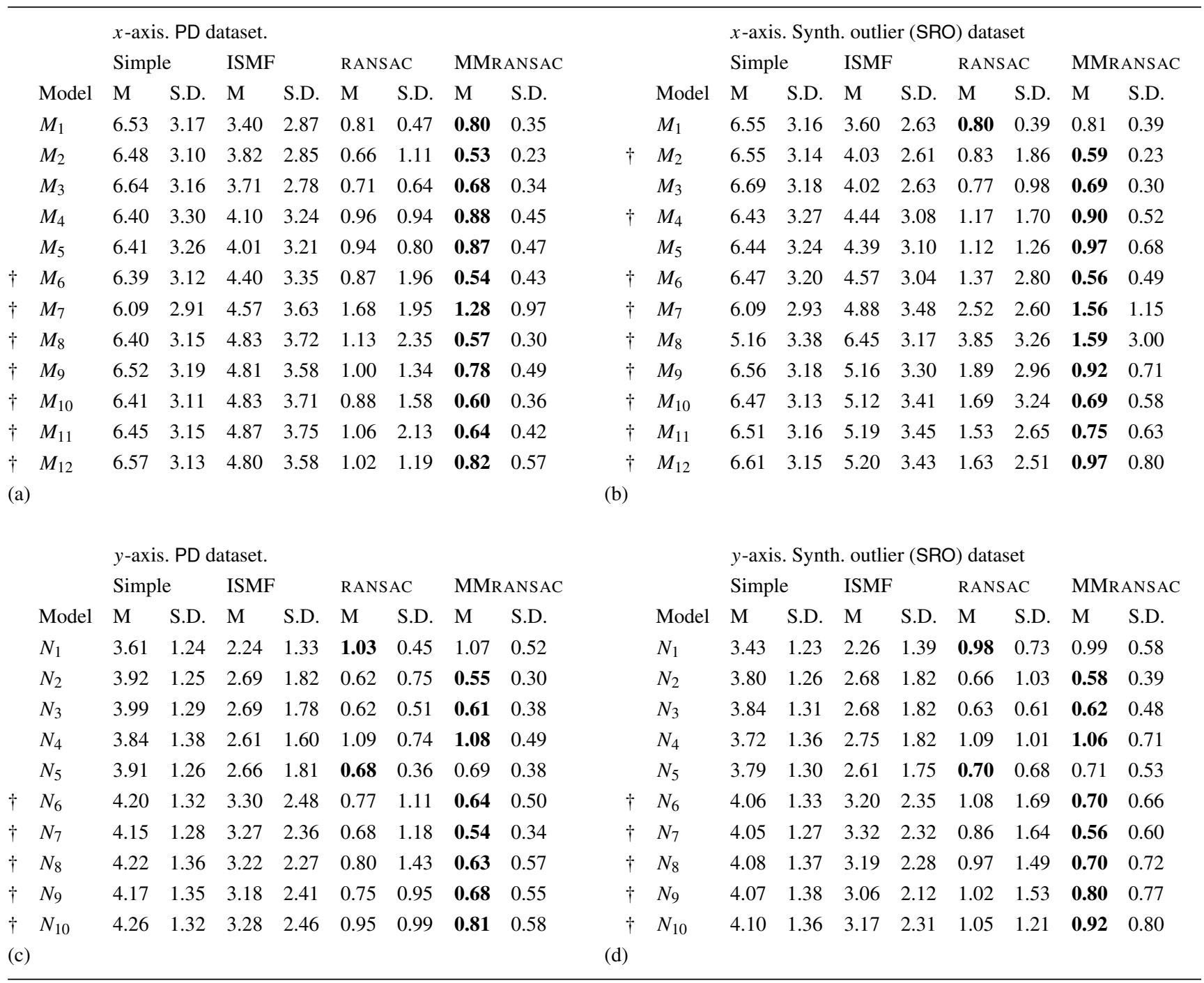

(a)(b) statistics for the PD dataset (b)(d) statistics for the PD dataset with a synthetic outlier. Best results are in bold. Dagger symbol $\dagger$ indicates significant differences between the RANSAC and MMRANSAC approaches 
Table 5 Statistics for the model selection experiment

\begin{tabular}{|c|c|c|c|c|c|c|c|c|c|c|c|}
\hline & & \multicolumn{2}{|l|}{ AIC } & \multicolumn{2}{|l|}{$\mathrm{AICc}$} & \multicolumn{2}{|l|}{ RAIC } & \multicolumn{2}{|l|}{ KIC } & \multicolumn{2}{|l|}{$\mathrm{KICc}$} \\
\hline & & M & S.D. & M & S.D. & M & S.D. & M & S.D. & M & S.D. \\
\hline \multirow[t]{3}{*}{ PD } & $x$-axis & 0.519 & 0.194 & 0.545 & 0.220 & 0.795 & 0.359 & 0.524 & 0.223 & 0.683 & 0.306 \\
\hline & $y$-axis & 0.503 & 0.258 & 0.529 & 0.257 & 0.805 & 0.466 & 0.510 & 0.259 & 0.726 & 0.365 \\
\hline & total & 0.511 & 0.228 & 0.537 & 0.239 & 0.800 & 0.416 & 0.517 & 0.242 & 0.705 & 0.337 \\
\hline \multirow[t]{3}{*}{ SRO } & $x$-axis & 0.566 & 0.303 & 0.585 & 0.275 & 0.820 & 0.404 & 0.569 & 0.298 & 0.706 & 0.291 \\
\hline & $y$-axis & 0.512 & 0.306 & 0.539 & 0.307 & 0.893 & 0.532 & 0.513 & 0.298 & 0.721 & 0.405 \\
\hline & total & 0.539 & 0.305 & 0.562 & 0.291 & 0.857 & 0.472 & 0.541 & 0.298 & 0.714 & 0.352 \\
\hline
\end{tabular}

Best results are in bold

Experiments Experiments were carried out in two phases:

- Parameter estimation. The objective of this experiment is to contrast the angular error of different parameter estimation strategies. Model parameters were estimated for each calibration set, and for each polynomial model by using the different estimation strategies. The lower quartile of absolute angular errors is used to calculate the log likelihood in the proposed approach.

- Model selection. Once the best parameter strategy was selected; the next research question is: which is the best model selector if we are interested in minimizing errors?

To answer this question, for each calibration set we proceeded as follows: a calibration using algorithm 1 was performed; then, the average of absolute angular errors for testing points was calculated and associated to each strategy.

Data analysis One-way ANOVA and Tamhane's T2 for pairwise comparisons tests were performed with the IBM SPSS Statistics 23 software. The level of statistical significance was set at $p<0.05$. In the experiments, we use the first quartile to quantify the resulting errors. Angular errors are expressed in degrees.

\section{Results and discussion}

Summary statistics for the first quartile angular error for $x$ - and $y$-axis are shown in Table 4. As shown in bold, both the RANSAC and MMRANSAC approaches reduce significantly the absolute angular (horizontal and vertical) errors in comparison with the ISMF approach. In general, there are no significant differences between RANSAC and the proposed approach for models with few coefficients (less than four). But, the MMRANSAC approach has significantly better results than RANSAC for models with four coefficients.
Results of the second test, "Model selection", are shown in Table 5. AIC and KIC criteria give the best results in average.

The model selection phase (line 4, algorithm 1) just improves marginally the average error compared to choosing the true single model; but this approach also reduces the variation in the calibration (reduction of the standard deviation).

Tests also show that some of the best results can be obtained with models of four parameters; e.g., model $M_{6}$. But, increasing the number of parameters could introduce some difficulties when the data contains outliers; e.g., the model $M_{8}$ gives one of the best results for the PD dataset (Table 5b) but it is the worst for the SO dataset (Table 5b). In this sense, the model selection phase is a "safer" way to face outliers.

The proposed approach is especially useful when there is no prior knowledge on the best model for the data.

Tested polynomial models shown in Table 2 were chosen instead of the exhaustive set (i.e., the power set of terms) to avoid sharp errors that may cause confusion.

\section{Conclusions and further work}

This paper proposes the MMRANSAC approach for robust Eye Tracker Calibration; tests on a publicly available dataset showed that this approach reduces significantly the angular absolute median errors in comparison with the conventional approach (that consists of removing initial measurements and taking the median value for each calibration point). We argue that the proposed approach is especially useful when a calibration set without outliers is hard to obtain (e.g., studies with infants), or when the best polynomial model is unknown for a specific device.

This paper also presents an approach that enables using conventional model selection statistics even in the presence of outliers. To avoid a boring calibration stage, e.g., when the user observes the predefined pattern. We envisage a 
continuous auto-calibration technique that take advantage of different mechanics of commercial video games.

Open Practices Statement The source code of this paper is available at https://github.com/albertlaar/mmransac.

\section{References}

Bernardo, J. M., et al. (1976). Psi (digamma) function. Applied Statistics, 25(3), 315-317.

Blignaut, P. (2016). Idiosyncratic feature-based gaze mapping. Journal of Eye Movement Research, 9(3).

Burnham, K. P., \& Anderson, D. R. (2003). Model selection and multimodel inference: A practical information-theoretic approach. Springer Science \& Business Media.

Cavanaugh, J. E., et al. (1997). Unifying the derivations for the Akaike and corrected Akaike information criteria. Statistics \& Probability Letters, 33(2), 201-208.

Cerrolaza, J. J., Villanueva, A., \& Cabeza, R. (2008). Taxonomic study of polynomial regressions applied to the calibration of videooculographic systems. In Proceedings of the 2008 symposium on eye tracking research \& applications, (pp. 259-266): ACM.

Cetin, M. C., \& Erar, A. (2002). Variable selection with Akaike information criteria: A comparative study. Hacettepe Journal of Mathematics and Statistics, 31, 89-97.

Cherif, Z. R., Nait-Ali, A., Motsch, J. F., \& Krebs, M. O. (2002). An adaptive calibration of an infrared light device used for gaze tracking. In IMTC/2002. Proceedings of the 19th IEEE Instrumentation and Measurement Technology Conference. vol. 2, (Vol. 2, pp. 1029-1033).

Drewes, J., Zhu, W., Hu, Y., \& Hu, X. (2014). Smaller is better: Drift in gaze measurements due to pupil dynamics. PloS one, 9(10).

Fischler, M. A., \& Bolles, R. C. (1981). Random sample consensus: A paradigm for model fitting with applications to image analysis and automated cartography. Communications of the ACM, 24(6), 381-395.

Gibaldi, A., Vanegas, M., Bex, P. J., \& Maiello, G. (2017). Evaluation of the Tobii EyeX Eye tracking controller and MATLAB toolkit for research. Behavior Research Methods, 49(3), 923-946.

Goldberg, J. H., \& Wichansky, A. M. (2003). Eye tracking in usability evaluation: A practitioner's guide. In The Mind's Eye, (pp. 493516): Elsevier.

Hansen, D. W., Agustin, J. S., \& Villanueva, A. (2010). Homography normalization for robust gaze estimation in uncalibrated setups. In Proceedings of the 2010 symposium on eye-tracking research \& applications, (pp. 13-20): ACM.
Hennessey, C., Noureddin, B., \& Lawrence, P. (2008). Fixation precision in high-speed noncontact eye-gaze tracking. IEEE Transactions on Systems, Man, and Cybernetics, Part B (Cybernetics), 38(2), 289-298.

Kasprowski, P., Harezlak, K., \& Stasch, M. (2014). Guidelines for the eye tracker calibration using points of regard. In Information Technologies in Biomedicine, (Vol. 4, pp. 225-236): Springer.

Krafka, K., Khosla, A., Kellnhofer, P., Kannan, H., Bhandarkar, S., Matusik, W., \& Torralba, A. (2016). Eye tracking for everyone. In Proceedings of the IEEE conference on computer vision and pattern recognition, (pp. 2176-2184).

Liu, Y., Emery, R., Chakrabarti, D., Burgard, W., \& Thrun, S. (2001). Using EM to learn 3D models of indoor environments with mobile robots. In ICML, (Vol. 1, pp. 329-336).

Majaranta, P., \& Bulling, A. (2014). Eye tracking and eyebased human-computer interaction. In Advances in physiological computing, (pp. 39-65): Springer.

Martinez, F., Carbone, A., \& Pissaloux, E. (2012). Gaze estimation using local features and non-linear regression. In IEEE International conference on image processing (ICIP), (pp. 1961-1964): IEEE.

Ohno, T., \& Mukawa, N. (2004). A free-head, simple calibration, gaze tracking system that enables gaze-based interaction. In Proceedings of the 2004 symposium on eye tracking research \& applications, (pp. 115-122): ACM.

Pomplun, M., Velichkovsky, B., \& Ritter, H. (1994). An artificial neural network for high precision eye movement tracking. In Annual conference on artificial intelligence, (pp. 63-69): Springer.

Raguram, R., Frahm, J.-M., \& Pollefeys, M. (2008). A comparative analysis of RANSAC techniques leading to adaptive real-time random sample consensus. In European conference on computer vision, (pp. 500-513): Springer.

Seghouane, A.-K., \& Bekara, M. (2004). A small sample model selection criterion based on Kullback's symmetric divergence. IEEE Transactions on Signal Processing, 52(12), 33143323.

SR Research Ltd. (2010). Eyelink1000 user manual. Version 1.5.0.

Zhu, Z., \& Ji, Q. (2005). Eye gaze tracking under natural head movements. In IEEE Computer society conference on computer vision and pattern recognition, 2005. CVPR 2005, (Vol. 1, pp. 918-923): IEEE.

Zhu, Z., Ji, Q., et al. ( 2007). Novel eye gaze tracking techniques under natural head movement. IEEE Transactions on Biomedical Engineering, 54(12), 2246.

Publisher's note Springer Nature remains neutral with regard to jurisdictional claims in published maps and institutional affiliations. 